QCD Evolution Workshop: From Collinear to Non-Collinear Case International Journal of Modern Physics: Conference Series Vol. 4 (2011) 190-199

(C) World Scientific Publishing Company

DOI: $10.1142 /$ S2010194511001693

\title{
PQCD ANALYSIS OF PARTON-HADRON DUALITY
}

\author{
SIMONETTA LIUTI \\ Department of Physics, University of Virginia, $382 \mathrm{McCormick} R$ d. \\ Charlottesville, VA 22904, USA \\ sl4y@virginia.edu
}

We propose an extraction of the running coupling constant of QCD in the infrared region from experimental data on deep inelastic inclusive scattering at Bjorken $x \rightarrow 1$. We first attempt a perturbative fit of the data that extends NLO PQCD evolution to large $x$ values and final state invariant mass, $W$, in the resonance region. We include both target mass corrections and large $x$ resummation effects. These effects are of order $\mathcal{O}\left(1 / Q^{2}\right)$, and they improve the agreement with the $Q^{2}$ dependence of the data. Standard analyses require the presence of additional power corrections, or dynamical higher twists, to achieve a fully quantitative fit. Our analysis, however, is regulated by the value of the strong coupling in the infrared region that enters through large $x$ resummation effects, and that can suppress, or absorb, higher twist effects. Large $x$ data therefore indirectly provide a measurement of this quantity that can be compared to extractions from other observables.

Keywords: Nucleon structure functions; momentum transfer dependence; quantum chromodynamics: perturbation theory; numerical calculations: interpretation of experiments.

PACS Nos.: 25.30.Dh, 25.30.F, 12.38.Bx, 12.38.Cyh

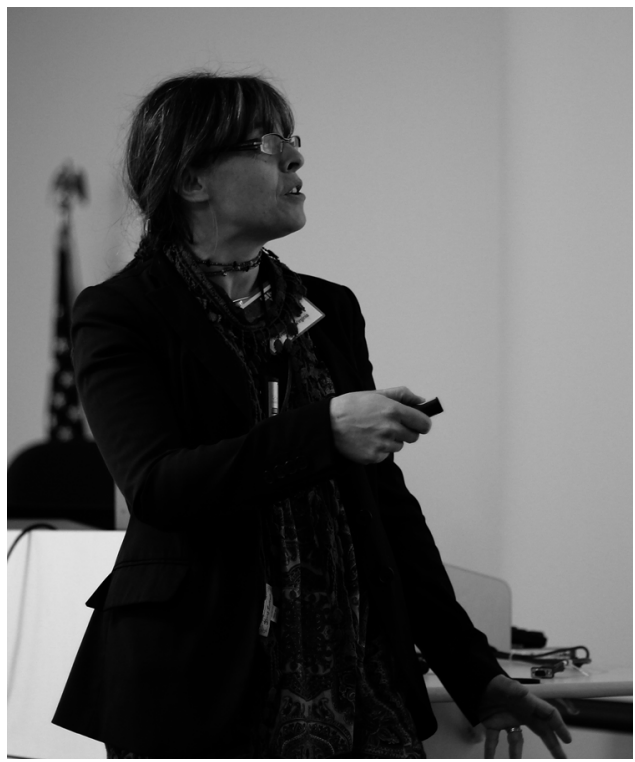




\section{Introduction}

The main goal of QCD is to describe the structure of hadrons in terms of its fundamental degrees of freedom given by the quarks and gluons, or partons. Hadrons are observable in both the initial and final stages of a hard process while the existence and properties of partons are inferred only indirectly. Because of the smallness of the running coupling constant of QCD at large enough momentum transfer squared, $Q^{2}$, or equivalently at short distances $\mathcal{O}\left(1 / \sqrt{Q^{2}}\right)$, a hard probe sees hadrons as composed of "free" quarks and gluons carrying fractions $x$ of the hadron's Light Cone (LC) momentum, with given probability distributions. Factorization theorems regulate this fundamental property of the theory by allowing for the short distance behavior at a given scale, $Q^{2}$, to be evaluated separately from the long distance contribution which is identified with the Parton Distribution Functions (PDFs) ${ }^{1}$. As increasingly shorter distances are probed the PDFs shape in $x$ changes due to radiative processes, according to a pattern which is calculable with high accuracy within Perturbative QCD (PQCD) ${ }^{2}$.

Although the perturbative phases of a hard collision are clearly distinct from the non-perturbative stage that gives rise to hadron structure, experimental observations indicate that such non-perturbative stage appears to be almost ubiquitously pre-determined by - or to be keeping track of - the perturbative mechanism. This concept goes under the name of parton-hadron duality. Parton-hadron duality implies in the most extreme case, that hadronic observables are replaced by calculable partonic ones with little more going into the hadronic formation phase of the process. In this respect, duality can be seen as a phenomenological manifestation of the non-perturbative to perturbative transition in QCD. A full explanation for its onset is however still lacking.

As an example in this contribution we will focus on Bloom Gilman (BG) duality ${ }^{3}$, observed in DIS. Here the relevant kinematical variables are: $x=Q^{2} / 2 M \nu(M$ being the proton mass and $\nu$ the energy transfer in the lab system), the four-momentum transfer, $Q^{2}$, and the invariant mass for the proton, $P$, and virtual photon, $q$, system, $W^{2}=(P+q)^{2}\left(W^{2}=Q^{2}(1 / x-1)+M^{2}\right)$. For large values of Bjorken $x \geq 0.5$, and $Q^{2}$ in the multi-GeV region, one has $W^{2} \leq 5 \mathrm{GeV}^{2}$, i.e. the cross section is dominated by resonance formation. While it is impossible to reconstruct the detailed structure of the proton's resonances, these remarkably follow the PQCD predictions when averaged over $x$ (see e.g. Ref.[4] for a review). Although BG duality was observed at the inception of QCD, quantitative analyses could be attempted only more recently, having at disposal the extensive, high precision data from Jefferson $\mathrm{Lab}^{4}$. Such analyses fall into two main categories:

i) Quark model/symmetries-based, Refs. $[5,6,7,8]$. These analyze the leading order matrix elements for the quark proton scattering subprocess and study the conditions under which the DIS cross section which is proportional to the incoherent sum of the squares of the constituent charges, can in average connect smoothly to the 
resonance production portion of the cross section which is given by the square of the coherent sum of the constituent charges. This connection is regulated by Parity transformations. It was, in fact, shown how the excitation of resonance states of opposite Parity interferes destructively in all cases except for the leading twist. No account of perturbative processes enters this model.

ii) PQCD-based, Refs. $[9,10,11]$. This approach claims that only after a complete perturbative QCD analysis is performed can one define duality by quantitatively establishing whether, and to what extent, this phenomenon is responsible for the apparent cancellation of multiparton correlations. It was noticed that the extension of NLO PQCD evolution to large $x$ must include large $x$ resummation effects. The overall effect of these is to shift the scale at which $\alpha_{S}$ is calculated to lower values, with increasing $x$. Standard analyses require the presence of additional power corrections, or dynamical higher twists, to achieve a fully quantitative fit. Our analysis, however, being regulated by the value of the QCD running coupling in the infrared region that enters through large $x$ resummation effects, brings to a suppression of higher twist effects. These get, in fact, absorbed in the coupling's infrared behavior.

Although these two approaches have been considered so far complementary to each other, a unified description might derive through the definition of the effective coupling $^{12}$.

This paper is organized as follows. In Section 2 we present an overview of the large $x$ data, and discuss a few aspects of the evolution mechanism for DIS at large $x$ where two scales related to the invariant mass and to the four-momentum transfer, are simultaneously present. We then illustrate in Section 3 the connection between large $x$ data and the coupling in the infrared region: we argue that once the range of validity of parton-hadron duality is defined quantitatively, a precise PQCD analysis at large $x$ would open up the possibility of extracting the strong coupling constant at low scale. In Section 4 we draw our conclusions

\section{Analysis of Large $x$ Data}

Our studies of the physical origin of duality, and of its impact on our understanding of the nucleon's structure started a few years ago when we set up a program to quantitatively extract the scale dependence of the large $x$ Jlab Hall $\mathrm{C}$ data $^{13}$. In the analysis performed in Refs. $[9,10]$ we addressed several effects that have a large impact at large $x$, namely Target Mass Corrections (TMCs), large $x$ resummation effects, and higher twists. This work was then completed, and extended to polarized data in Ref. [11]. An important point emerged from Refs.[9, 10, 11] that a deeper understanding was needed of those aspects unique to the large $x$ perturbative QCD analysis.

High precision inclusive unpolarized electron-nucleon scattering data on both hydrogen and deuterium targets from Jefferson Lab are available to date in the large $x$, multi-GeV regime (seeRef.[16] and references therein). Because of the precision 
of the data one should now be able to distinguish among different sources of scaling violations affecting the structure functions in addition to standard NLO evolution,

- Target Mass Corrections (TMC),

- Large $x$ Resummation Effects (LxR)

- Nuclear Effects

- Dynamical Higher Twists (HTs),

- Impact of Next-to-Next-to-Leading-Order (NNLO) perturbative evolution.

All of the effects above can be extracted with an associated theoretical error. It is in fact well known that their evaluation is model dependent. Recent studies, however, have been directed at determining more precisely both the origin and size of the associated theoretical error. Recent analyses have been taking into account, so far, some but not all of the effects listed above ${ }^{17}$.

\subsection{Unpolarized structure function}

The inclusive DIS cross section of unpolarized electrons off an unpolarized proton is written in terms of the two structure functions $F_{2}$ and $F_{1}$,

$$
\frac{d^{2} \sigma}{d x d y}=\frac{4 \pi \alpha^{2}}{Q^{2} x y}\left[\left(1-y-\frac{(M x y)^{2}}{Q^{2}}\right) F_{2}+y^{2} x F_{1}\right],
$$

with $y=\nu / \epsilon_{1}, \epsilon_{1}$ being the initial electron energy. The structure functions are related by the equation

$$
F_{1}=F_{2}\left(1+\gamma^{2}\right) /(2 x(1+R)),
$$

where $\gamma^{2}=4 M^{2} x^{2} / Q^{2}$, and $R$ is ratio of the longitudinal to transverse virtual photo-absorption cross sections. In QCD, $F_{2}$ is expanded in series of inverse powers of $Q^{2}$, obtained by ordering the matrix elements in the DIS process by increasing twist $\tau$, which is equal to their dimension minus spin

$$
\begin{aligned}
F_{2}\left(x, Q^{2}\right) & =F_{2}^{L T}\left(x, Q^{2}\right)+\frac{H(x)}{Q^{2}}+\mathcal{O}\left(\frac{1}{Q^{4}}\right) \\
& \simeq F_{2}^{L T}\left(x, Q^{2}\right)\left(1+\frac{C(x)}{Q^{2}}\right)+\mathcal{O}\left(\frac{1}{Q^{4}}\right)
\end{aligned}
$$

The first term is the leading twist (LT), with $\tau=2$. The terms of order $1 / Q^{\tau-2}$, $\tau \geq 4$, in Eq.(3) are the higher order terms, generally referred to as higher twists ${ }^{1}$.

\section{Target Mass Corrections}

TMCs are included in $F_{2}^{L T}$. For $Q^{2} \geq 1 \mathrm{GeV}^{2}$, TMCs can be taken into account through the following expansion ${ }^{18}$

$$
F_{2}^{L T(T M C)}\left(x, Q^{2}\right)=\frac{x^{2}}{\xi^{2} \gamma^{3}} F_{2}^{\infty}\left(\xi, Q^{2}\right)+6 \frac{x^{3} M^{2}}{Q^{2} \gamma^{4}} \int_{\xi}^{1} \frac{d \xi^{\prime}}{\xi^{\prime 2}} F_{2}^{\infty}\left(\xi^{\prime}, Q^{2}\right)
$$


where $F_{2}^{\infty}$ is the structure function in the absence of TMCs. A more recent analysis ${ }^{19}$ re-examined TMCs within the collinear factorization approach of [20] in order to address the longstanding question of the unphysical behavior in the threshold region of Eq.(4). This originates from the fact that as $x \rightarrow 1$, one obtains a $Q^{2}$-dependent threshold, namely $F_{2}\left(\xi, Q^{2}\right)=0$ for $\xi>\xi_{\max }=2 / 1+\sqrt{1+4 M^{2} / Q^{2}}$, therefore rendering $F_{2}$ undefinable as $Q^{2}$ varies (see discussion in Ref.[21]). In the formalism of Ref.[19], TMCs, applied to the helicity dependent structure functions read

$$
F_{T}^{L T(T M C)}\left(x, Q^{2}\right)=\int_{m_{\pi}^{2}}^{\frac{1-x}{x} Q^{2}} d m_{J}^{2} \rho\left(m_{J}^{2}\right) F_{T}^{\infty}\left[\xi\left(1+\frac{m_{J}^{2}}{Q^{2}}\right), Q^{2}\right]
$$

where $F_{T} \equiv F_{1}$. The final quark is assumed to hadronize into a jet of mass $m_{J}$ with a a process dependent distribution/smearing function $\rho\left(m_{J}^{2}\right)$. In our extraction we take the perspective that the evaluation of TMCs is always associated with the evaluation of HTs - TMCs should in principle be applied also to HTs - in an inseparable way. Therefore we consistently keep terms of $\mathcal{O}\left(1 / Q^{4}\right)^{11,22}$, whether in the formalism/prescription of Ref.[18] or of Ref.[19]. $H\left(x, Q^{2}\right)$, then, represents the "genuine" HT correction that involves interactions between the struck parton and the spectators or, formally, multi-parton correlation functions.

\section{Threshold Resummation}

In order to understand the nature of the remaining $Q^{2}$ dependence that cannot be described by NLO pQCD evolution, we also include the effect of threshold resummation, or Large $x$ Resummation (LxR). LxR effects arise formally from terms containing powers of $\ln (1-z), z$ being the longitudinal variable in the evolution equations, that are present in the Wilson coefficient functions $C(z)$. Below we write schematically how the latter relate the parton distributions to e.g. the structure function $F_{2}$,

$$
F_{2}^{L T}\left(x, Q^{2}\right)=\frac{\alpha_{s}}{2 \pi} \sum_{q} \int_{x}^{1} d z C(z) q\left(x / z, Q^{2}\right)
$$

where we have considered only the non-singlet (NS) contribution to $F_{2}$ since only valence quarks distributions are relevant in our kinematics. The logarithmic terms in $C(z)$ become very large at large $x$, and they need to be resummed to all orders in $\alpha_{S}$. Resummation was first introduced by linking this issue to the definition of the correct kinematical variable that determines the phase space for the radiation of gluons at large $x$. This was found to be $\widetilde{W}^{2}=Q^{2}(1-z) / z$, instead of $Q^{2}[23,24]$. As a result, the argument of the strong coupling constant becomes $z$-dependent: $\alpha_{S}\left(Q^{2}\right) \rightarrow \alpha_{S}\left(Q^{2}(1-z) / z\right)[25,26]$. In this procedure, however, an ambiguity is introduced, related to the need of continuing the value of $\alpha_{S}$ for low values of its argument, i.e. for $z \rightarrow 1$ [27]. Although on one side, the size of this ambiguity could be of the same order of the HT corrections and, therefore, a source of theoretical 
error, on the other by performing an accurate analysis such as the one proposed here, one can extract $\alpha_{S}$ for values of the scale in the infrared region. We address this point in more detail in the next Section.

\section{Nuclear Effects}

Theoretical uncertainties in the deuteron are taken routinely into account, and are expected to be in sufficient control (see Ref. [28] and references therein). Uncertainties arise mainly from

i) Different models of the so called nuclear EMC effect;

ii) Different deuteron wave functions derived from currently available NN potentials, giving rise to different amounts of high momentum components;

iii) The interplay between nucleon off-shellness and TMC in nuclei.

Finally, we did not consider NNLO calculations, these are not expected to alter substantially our extraction since, differently from what seen originally in the case of $F_{3}$, these have been proven to give a relatively small contribution to $F_{2}$.

Once all of the above effects have been subtracted from the data, and assuming the validity of the twist expansion, Eq.(3) in this region, one can interpret more reliably any remaining discrepancy in terms of HTs. Since we extend our $x$-dependent analysis to the resonance region we consider the following integrated quantities

$$
I^{\mathrm{res}}\left(\langle x\rangle, Q^{2}\right)=\int_{x_{\min }}^{x_{\max }} F_{2}^{\mathrm{res}}\left(x, Q^{2}\right) d x
$$

where $F_{2}^{\text {res }}$ is evaluated using the experimental data in the resonance region. For each $Q^{2}$ value: $x_{\min }=Q^{2} /\left(Q^{2}+W_{\max }^{2}-M^{2}\right)$, and $x_{\max }=Q^{2} /\left(Q^{2}+W_{\min }^{2}-M^{2}\right)$, where $W_{\min }$ and $W_{\max }$ delimit the resonance region, and $\langle x\rangle$ is the average value of $x$ for each kinematics. This procedure replaces a strict point by point in $x$, analysis.

Typical results from the analysis outlined above are plotted in Fig. 2.1, where we show the HT coefficient defined from Eq.(3) as

$$
R^{L T} \equiv C(x)=Q^{2}\left[F_{2}\left(x, Q^{2}\right) / F_{2}^{L T}\left(x, Q^{2}\right)-1\right]
$$

The error in the figure is from the experimental data. No theoretical uncertainty was included. However, our results clearly show that the combined effects of TMCs and LxR substantially reduce $C(x)$. We take this as illustrative of the accomplishments one can expect from the analysis we suggest in this contribution. Essential features that emerge are the interplay between the values of $\alpha_{S}\left(M_{Z}^{2}\right)$ and the HTs, the relevance of TMCs, and, most importantly, the need to define $\alpha_{S}$ in the infrared region. All of these features can affect the central values of the HTs reported in Fig. 1. 


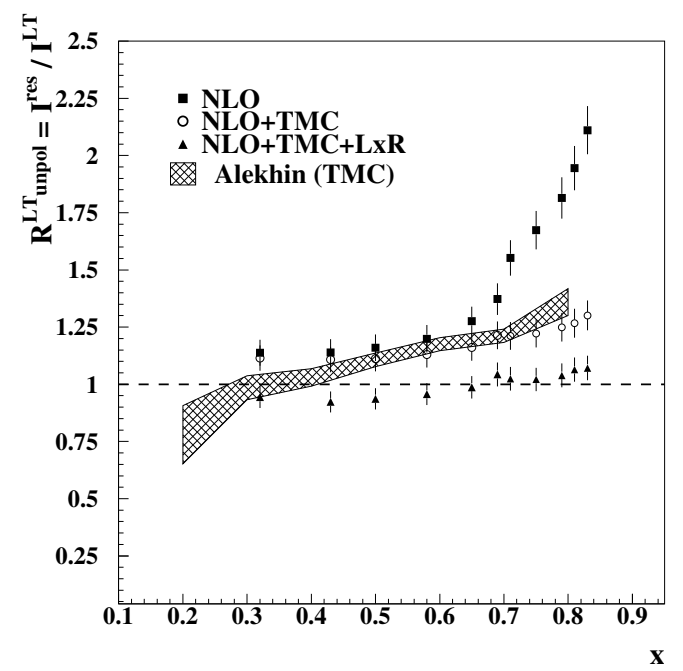

Fig. 1. HT coefficients extracted in the resonance region according to the procedure described in the text. HT extracted with only the NLO calculation (squares); the effect subtracting TMC (open circles); the effect of subtracting both TMC and LxR (triangles). Shown for comparison are the values obtained from the coefficient $H$ obtained in Ref. [29] using DIS data and including the effect of TMC. Adapted from Ref. [11].

\section{Strong Coupling Constant at High $x$}

We now discuss in more detail the working of threshold resummation, and its possible impact on the analysis of $F_{2}$ at large $x$ [25]. Starting from NLO, the coefficient, $C(z)$ in Eq.(6) is dominated at large $x$ by terms proportional to $\left[\alpha_{S}\left(Q^{2}\right) \ln (1-z)\right]^{n}$ which need to be resummed in the perturbative series. The physical origin of these terms is in the phase space for the contribution of gluons emission to evolution, which become soft as $x \rightarrow 1$. A mismatch in the cancellation with the virtual gluons contributions ensues. If, however, one carefully evaluates the kinematics for gluon emission at large $x$ within a quark-parton model view, one obtains ${ }^{23}$

$$
q\left(x, Q^{2}\right)=q\left(x, Q_{o}^{2}\right)+\int_{Q_{o}^{2}}^{\widetilde{W}^{2}} \frac{d k_{\perp}^{2}}{k_{\perp}^{2}} \frac{\alpha_{S}\left(k_{\perp}^{2}\right)}{2 \pi} \int_{x}^{1} \frac{d z}{z} P_{q q}\left(\frac{x}{z}, \alpha_{S}\right) q\left(z, k_{\perp}^{2}\right),
$$

where $Q_{o}^{2}$ is an arbitrary initial scale, and $\widetilde{W}^{2}=Q^{2}(1-z) / z$ is the maximum $k_{\perp}^{2}$ in the virtual photon-quark center of mass system, appearing in the ladder graphs that define the leading $\log$ result. The resulting phase space is shown for different $Q^{2}$ values in Fig. 2. The reduction of the allowed $k_{\perp}^{2}$ results in a simultaneous shift in the argument of $\alpha_{S} \rightarrow \alpha_{S}\left(Q^{2} /(1-z) / z\right)$, and a cancellation of the $\alpha_{S}\left(Q^{2}\right) \ln (1-z)$ divergence in the NLO coefficient function. As a consequence of rescaling the argument of $\alpha_{S}$ one has to consider its continuation into the infrared region ${ }^{11,26}$. The left panels of Fig. 3 display the results for $\alpha_{S}$ used in our analysis for different values of $Q^{2}$. We also show, on the right, the extracted value of the effective $\alpha_{S}$ from the 


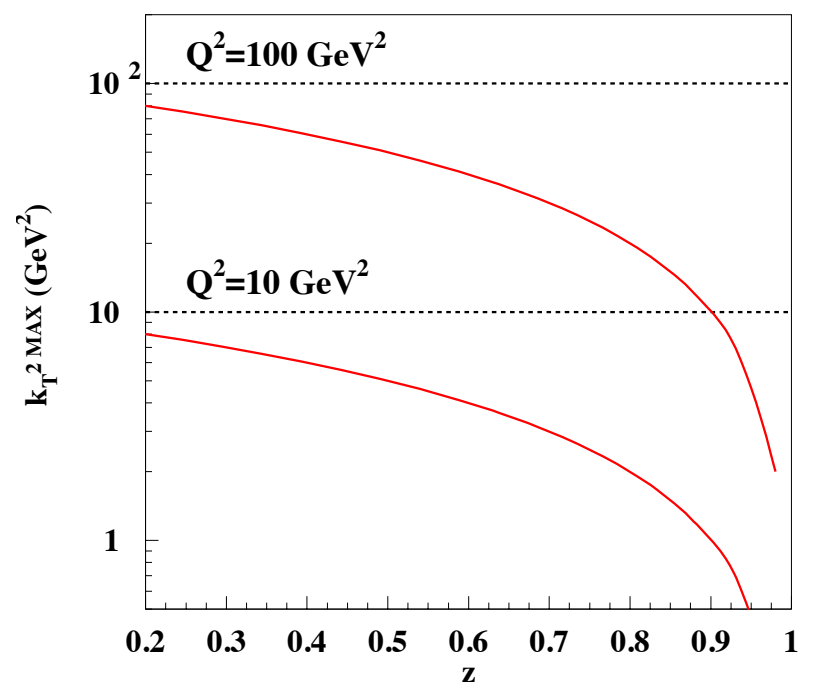

Fig. 2. Phase space in the evolution of the NS component. The dotted lines are for $k_{T, \max }^{2}=Q^{2}$, for $Q^{2}=10,100 \mathrm{GeV}^{2}$. The full lines represent the upper limit $k_{T, \max }^{2}=Q^{2}(1-z) / z$ for $Q^{2}=$ $10,100 \mathrm{GeV}^{2}$. In this case, one can see a clear reduction of the allowed $k_{T}$ at large $z$.

GDH sum rule. We therefore suggest large $x$ evolution in DIS as yet another way of defining an effective coupling constant at low values of the scale. A more quantitative analysis to relate different types of measurements, and to study in depth the possible process dependence of $\alpha_{S}$ is in progress ${ }^{12}$.

\section{Conclusions}

In conclusion, we believe there is a much richer structure to the scale dependence of the nucleon's distribution functions that persists behind the apparent cancellation among higher twist terms. We started uncovering this structure in the initial work of Refs. $[9,11]$. Our analysis opens up the possibility of extracting the effective strong coupling at low scale, and to connect it to extractions from different processes, namely Refs.[14, 15] and Ref.[30]. While on one side this points at the fact that PQCD provides an essential framework for understanding the working of duality, a possible connection with the model of Refs. $[5,6,7,8]$ is envisaged through the definition of effective charge ${ }^{12}$. 

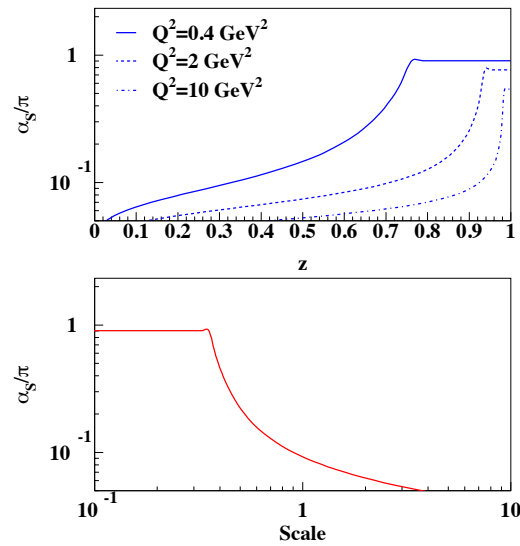

Fig. 3. Left panel: $\alpha_{S} / \pi$ extracted from the analysis of the large $x$ data discussed in the text, and plotted vs. $z$, Eq. (6) (upper panel), and vs. $\widetilde{W}=\sqrt{Q^{2}(1-z) / z}$ (lower panel). For comparison we show the extraction from Ref.[14] using Jefferson Lab data at $Q^{2}=0.7-1.1 \mathrm{GeV}^{2}$.

\section{Acknowledgments}

This work is supported by the U.S. Department of Energy grant no. DE-FG0201ER41200. Many discussions with J.P. Chen, A. Courtoy, A. Deur and V. Vento are gratefully acknowledged.

\section{References}

1. CTEQ Collaboration "The Handbook of Perturbative QCD", Rev. of Mod. Phys., 67, 157 (1995)

2. S. Alekhin, J. Blumlein, S. Klein et al., Phys. Rev. D81, 014032 (2010).

3. E.D. Bloom and F.J. Gilman, Phys. Rev. Lett. 25, 1140 (1970); Phys. Rev. D4, 2901(1971).

4. W. Melnitchouk, R. Ent, C. Keppel, Phys. Rept. 406, 127-301 (2005).

5. F. E. Close, N. Isgur, Phys. Lett. B509, 81-86 (2001).

6. F. E. Close, W. Melnitchouk, Phys. Rev. C68, 035210 (2003); ibid Phys. Rev. C79, $055202(2009)$.

7. S. Jeschonnek, J. W. Van Orden, Phys. Rev. D69, 054006 (2004).

8. Q. Zhao, F. E. Close, Phys. Rev. Lett. 91, 022004 (2003).

9. S. Liuti, R. Ent, C. E. Keppel et al., Phys. Rev. Lett. 89, 162001 (2002).

10. C. S. Armstrong, R. Ent, C. E. Keppel et al., Phys. Rev. D63, 094008 (2001).

11. N. Bianchi, A. Fantoni, S. Liuti, Phys. Rev. D69, 014505 (2004); ibid, AIP Conf. Proc. 747, 126-129 (2005); ibid S. Liuti, N. Bianchi, A. Fantoni, AIP Conf. Proc. 792, 253-256 (2005).

12. J.P. Chen, A. Courtoy, A. Deur, S. Liuti, and V. Vento, work in progress.

13. I. Niculescu et al., Phys. Rev. Lett. 85, 1186 (2000).

14. A. Deur, V. Burkert, J. P. Chen et al., Phys. Lett. B665, 349-351 (2008).

15. S. J. Brodsky, G. F. de Teramond, A. Deur, Phys. Rev. D81, 096010 (2010).

16. M. E. Christy, P. E. Bosted, Phys. Rev. C81, 055213 (2010).

17. A. Accardi, M. E. Christy, C. E. Keppel et al., Phys. Rev. D81, 034016 (2010). 
18. A. De Rujula, H. Georgi and H.D. Politzer, Phys. Lett. B64, 428 (1976); ibid Ann. Phys. 103, 315 (1977).

19. A. Accardi, J. -W. Qiu, JHEP 0807, 090 (2008).

20. J. C. Collins, T. C. Rogers, A. M. Stasto, Phys. Rev. D77, 085009 (2008).

21. I. Schienbein, V. A. Radescu, G. P. Zeller et al., J. Phys. G G35, 053101 (2008).

22. S. I. Alekhin, S. A. Kulagin, S. Liuti, Phys. Rev. D69, 114009 (2004).

23. S. J. Brodsky and G. P. Lepage, "Perturbative Quantum Chromodynamics," SLACPUB-2447.

24. D. Amati et al., Nucl. Phys. B173, 429 (1980).

25. R.G. Roberts, The Structure of the Proton, Cambridge University Press, (1993).

26. R. G. Roberts, Eur. Jour. of Phys. C10, 697 (1999).

27. M. R. Pennington and G. G. Ross, Phys. Lett. B102, 167 (1981).

28. S. A. Kulagin, R. Petti, Phys. Rev. C82, 054614 (2010).

29. S. I. Alekhin, V JHEP 0302, 015 (2003).

30. A. Courtoy, S. Scopetta and V. Vento, Eur. Phys. J. A 47, 49 (2011); A. Courtoy, arXiv:1107.4880 [hep-ph]. 